Cahiers
de la Recherche
sur les Droits

Cahiers de la recherche sur les droits fondamentaux

16 | 2018

Les partis politiques

\title{
Les politiques de rationalisation des financements partisans
}

Policies for Streamlining Partisan Fundraising

Yves Poirmeur

\section{(2) OpenEdition}

\section{Journals}

Édition électronique

URL : https://journals.openedition.org/crdf/305

DOI : $10.4000 /$ crdf.305

ISSN : 2264-1246

Éditeur

Presses universitaires de Caen

Édition imprimée

Date de publication : 16 novembre 2018

Pagination : 35-43

ISBN : 978-2-84133-901-3

ISSN : $1634-8842$

\section{Référence électronique}

Yves Poirmeur, "Les politiques de rationalisation des financements partisans », Cahiers de la recherche sur les droits fondamentaux [En ligne], 16 | 2018, mis en ligne le 16 novembre 2019, consulté le 15 novembre 2022. URL : http://journals.openedition.org/crdf/305; DOI : https://doi.org/10.4000/crdf. 305

Tous droits réservés 


\title{
Les politiques de rationalisation des financements partisans
}

\author{
Yves POIRMEUR \\ Professeur de science politique à l'université de Versailles Saint-Quentin-en-Yvelines \\ Centre de recherche Versailles Saint-Quentin institutions publiques (VIP)
}

I. La rationalisation de la condition économique des partis politiques

A. La construction d'une politique d'indépendance financière des partis

B. L'amélioration du dispositif d'encadrement, de contrôle et de sanctions

II. Les incidences du système de financement public

A. La soustraction des partis aux pressions financières

B. Les métamorphoses des partis

Longtemps oublié du droit français, le financement des partis politiques est devenu depuis une trentaine d'années un objet de politiques publiques. Les $\mathrm{III}^{\mathrm{e}}$ et $\mathrm{IV}^{\mathrm{e}}$ Républiques leur avaient laissé, en les ignorant ${ }^{1}$, une liberté presque complète, que la Constitution de la $\mathrm{V}^{\mathrm{e}}$ leur avait ensuite garantie $^{2}$. Les partis n'étaient donc soumis, qu'il s'agisse de leurs ressources ou de leurs dépenses, à aucune obligation spécifique, ce qui ne les exonérait pas de respecter le droit commun, comme l'a montré à la fin des années 1980 la multiplication des scandales politico-financiers liés à l'irrégularité des procédés de collecte de fonds auxquels ils recouraient pour se procurer de l'argent ${ }^{3}$. Pour sortir de ces affaires qui empoisonnaient la vie politique, le législateur a commencé à construire empiriquement, sans ordre ni méthode, un droit des financements partisans. Trois lois successives - 1988, 1990 et 1995 - ont été nécessaires pour en déterminer les orientations. La première a introduit un financement public $^{4}$; la deuxième a mis en place la Commission nationale des comptes de campagne et des financements politiques (CNCCFP) ${ }^{5}$; la troisième $\mathrm{a}$ interdit aux personnes morales de financer les partis et réglementé les dons ${ }^{6}$. Retouchée à maintes reprises ${ }^{7}$, au gré des intérêts

1. Voir R. Kraehe, Le financement des partis politiques, Paris, Presses universitaires de France, 1972, p. 16.

2. Art. 4 de la Constitution du 4 octobre 1958.

3. Sur ces pratiques occultes, voir J.-P. Camby, Le financement de la vie politique en France, Paris, Montchrestien, 1995 , p. 45 sq.

4. Loi $\mathrm{n}^{\circ}$ 88-227 du 11 mars 1988 relative à la transparence financière de la vie politique.

5. Elle a été créée par la loi nº 90-55 du 15 janvier 1990 relative à la limitation des dépenses électorales et à la clarification du financement des activités politiques.

6. Art. 16 de la loi no $95-65$ du 19 janvier 1995 relative au financement de la vie politique: «Les personnes morales à l'exception des partis ou groupements politiques ne peuvent contribuer au financement des partis ou groupements politiques, ni en consentant des dons, sous quelque forme que ce soit, à leurs associations de financement ou à leurs mandataires financiers, ni en leur fournissant des biens, services ou autres avantages directs ou indirects à des prix inférieurs à ceux qui sont habituellement pratiqués».

7. Art. 15 et 16 de la loi no $2013-907$ du 11 octobre 2013 relative à la transparence de la vie publique; art. 7 de la loi organique $n^{\circ} 2016-506$ du 25 avril 2016 de modernisation des règles applicables à l'élection présidentielle; art. 3 de la loi no 2016-508 du 25 avril 2016 de modernisation de diverses règles applicables aux élections; art. 5 à 9 de la loi no 2017-286 du 6 mars 2017 tendant à renforcer les obligations comptables des partis politiques et des candidats. 
politiques qui en ont altéré parfois la logique ${ }^{8}$, cette législation a acquis une certaine cohérence. Ce dispositif répond dans ses grandes lignes, aux prescriptions d'un référentiel normatif qui, progressivement enrichi, diversifie les finalités assignées à la réglementation des financements partisans et préside à son évolution. Dotant les formations politiques, grâce à un financement public, de ressources suffisantes au libre exercice de leurs activités et assurant par un système de contrôle une certaine transparence de l'origine et de l'emploi des fonds qu'elles recueillent, cette législation régulièrement améliorée a institué un régime de financement adapté au rôle indispensable qu'elles jouent dans la société démocratique, même s'il comporte encore des lacunes et des insuffisances que confirment de nouvelles affaires de financement irrégulier (I). Rendue nécessaire par l'obsolescence de modes de financement qui n'étaient plus viables, cette rationalisation du droit des financements partisans n'a fait qu'accompagner la mutation structurelle des partis qui, en France comme ailleurs en Europe ${ }^{9}$, deviennent des partis électoraux professionnels ${ }^{10}$. Accélérant cette conversion qui les éloigne des citoyens, elle est loin d'avoir atteint les objectifs qui lui étaient fixés de rétablir leur confiance dans la politique et d'encourager leur participation aux élections ${ }^{11}$, elle accompagne le changement de la forme parti ${ }^{12}$ et la mutation des stratégies d'influence des groupes d'intérêt (II).

\section{La rationalisation de la condition économique des partis politiques}

Entreprises de conquête ${ }^{13}$ et, en cas de succès, d'exercice du pouvoir politique, ayant besoin de se procurer pour ce faire l'argent nécessaire à la rémunération de leurs dirigeants et de leurs permanents, à la couverture de leurs charges de fonctionnement et au paiement des dépenses de campagne des candidats qu'ils présentent aux élections, les partis politiques sont profondément marqués par la façon dont ils obtiennent de l'argent et par l'importance de leurs ressources financières qui déterminent largement leurs capacités rétributives ${ }^{14}$. En France, où à partir de 1901 ils ont été régis par le droit minimaliste de la loi relative au contrat d'association ${ }^{15}$, complété ensuite par la loi du 10 janvier 1936 sur les groupes de combat et les milices privées, leur liberté de financement était entière. Cette liberté a permis l'éclosion, aux côtés des entreprises politiques individuelles que de grands notables ou de riches hommes d'affaires avaient toujours les moyens de constituer, mais qui peu à peu sortaient de l'histoire, de deux grands types d'organisations partisanes: des partis de cadres, tirant principalement leur argent des dons importants de sympathisants et de cotisations élevées d'adhérents peu nombreux disposant d'importantes ressources économiques et sociales; des partis de masses, l'obtenant de nombreux petits dons et cotisations, cherchant donc «à faire des adhérents [...] et à grossir leurs effectifs ${ }^{16}$ et accordant à cet effet une place considérable aux activités militantes. À quelque type qu'elles aient appartenu et quelle qu'ait été l'origine principale de leurs ressources, ces formations politiques pouvaient recevoir non seulement des dons d'entreprises, de groupes d'intérêt ou de mécènes français ou étrangers, mais encore, le cas échéant, en l'absence de tout contrôle de l'origine des fonds, d'États étrangers. Disposant de fonds secrets en argent liquide, le gouvernement pouvait aussi en faire profiter les formations politiques qui le soutenaient. Malgré les scandales suscités par le caractère trouble de ces financements ${ }^{17}$, ce n'est qu'après la Seconde Guerre mondiale que l'idée de les encadrer a été avancée, mais sans succès. Elle se heurtera ensuite à la consécration juridique de la liberté des partis politiques par la Constitution de 1958 dont l'article 4 dispose que

Les partis et groupements politiques concourent à l'expression du suffrage. Ils se forment et exercent leur activité librement. Ils doivent respecter les principes de la souveraineté nationale et de la démocratie ${ }^{18}$.

8. C'est ainsi, par exemple, qu'une « erreur » lors du vote de la loi du 11 octobre 2013 relative à la transparence de la vie publique avait malencontreusement conduit à supprimer les sanctions pénales qui étaient prévues lorsqu'un parti acceptait des dons de personnes morales autres que des partis. Elles ont été rétablies par la loi n ${ }^{\circ}$ 2015-1703 du 21 décembre 2015 visant à pénaliser l'acceptation par un parti politique d'un financement par une personne morale.

9. Voir S. E. Scarrow, « Parties without Members? Party Organization in a Changing Electoral Environment», in Parties without Partisans: Political Change in Advanced Industrial Democracies, R. J. Dalton, M. P. Wattenberg (dir.), New York, Oxford University Press, 2000, p. 79-101.

10. Voir A. Panebianco, Political Parties: Organization and Power, Cambridge, Cambridge University Press, 1988, p. 264.

11. Commission européenne pour la démocratie par le droit, Code de bonne conduite en matière de partis politiques, Venise, 12-13 décembre 2008 et Rapport explicatif, Venise, 13-14 mars 2009, $n^{\circ} 154$, p. 42.

12. Voir P. Pombeni, Introduction à l'histoire des partis politiques, Paris, Presses universitaires de France, 1992, p. 165.

13. Pour Max Weber, les partis sont «des sociations reposant sur un engagement (formellement) libre ayant pour but de procurer à leurs chefs le pouvoir au sein d'un groupe» (M. Weber, Économie et société, Paris, Pocket, 1995, t. I, p. 371).

14. Y. Poirmeur, Les partis politiques: $d u$ XIX $X^{e}$ au XXI siècle en France, Issy-les-Moulineaux, LGDJ, 2014, p. 61 sq. Les postes électifs et administratifs que les partis contrôlent dans l'État et ses démembrements sont, avec l'argent, des ressources essentielles pour rétribuer le militantisme; voir D. Gaxie, «Économie des partis et rétribution du militantisme», Revue française de science politique, $\mathrm{n}^{\circ} 1,1977$.

15. Voir Y. Poirmeur, D. Rosenberg, Droit des partis politiques, Paris, Ellipses, 2008.

16. M. Duverger, Les partis politiques, Paris, Seuil, 1981, p. 70.

17. Sur le scandale en 1924 du financement des candidats à la députation et de leur parti par la caisse électorale de l’Union des intérêts économiques, voir N. Tolini, Le financement des partis politiques, Paris, Dalloz, 2007, p. 46, note 67.

18. La loi constitutionnelle $n^{\circ}$ 99-569 du 8 juillet 1999 relative à l'égalité des femmes et des hommes lui a ajouté l'alinéa suivant: «Ils contribuent à la mise en œuvre du principe énoncé au second alinéa de l'article $1^{\text {er }}$ dans les conditions déterminées par la loi [égalité d'accès des femmes et des hommes aux mandats électoraux et aux fonctions électives]». Celle $n^{\circ}$ 2008-724 du 23 juillet 2008 de modernisation des institutions de la $V^{e}$ République lui a ajouté l'alinéa suivant: «La loi garantit les expressions pluralistes des opinions et la participation équitable des partis et groupements politiques à la vie démocratique de la Nation». 
Dans les années 1980, la multiplication des scandales politico-financiers et «l'inflation du coût de la démocratie ${ }^{19}$ poseront sous un jour nouveau le problème de la régulation des financements partisans en ajoutant à la vieille question du contrôle de l'origine de leurs fonds, celle de leur procurer suffisamment d'argent. Un référentiel normatif d'indépendance des partis à l'égard des influences financières des intérêts privés a ainsi été construit qui sous-tend la politique de régulation des financements partisans (A) et a permis progressivement la rationalisation de leurs financements légaux (B).

\section{A. La construction d'une politique d'indépendance financière des partis}

S’ils échouent à faire adopter un statut aux partis politiques, des projets constitutionnels émanant de partis et différentes propositions doctrinales dégagent, à la fin de la III ${ }^{\mathrm{e}}$ République ${ }^{20}$ et au début de la $\mathrm{IV}^{\mathrm{e}}$, plusieurs principes constitutifs du futur référentiel de la politique de rationalisation du financement des partis politiques ${ }^{21}$. Le premier répond aux inquiétudes soulevées, en période de guerre froide, par le financement de certains partis par des États étrangers et au souci de protéger l'indépendance nationale. Marcel Waline notant que la ligne du parti communiste était dictée de l'étranger préconise ainsi de nationaliser les partis:

[...] les finances de tout groupement politique devraient donc être soumises à un contrôle serré de la part d'experts comptables qui publieraient un rapport annuel; ce rapport, ou des extraits établissant l'origine des fonds, devraient être publiés dans la presse. [...] La sanction de l'opinion ne devrait pas suffire: tout parti convaincu d'avoir reçu des fonds d'un gouvernement étranger, ou d'un organisme de propagande ayant son siège à l'étranger, devrait être dissous par décision de justice motivée ${ }^{22}$.

La deuxième préoccupation est d'assurer un bon fonctionnement du système démocratique, qui comporte plusieurs facettes. Il serait nécessaire, d'abord, d'établir une transparence des financements partisans afin de permettre aux électeurs de faire un choix éclairé entre les candidats que les formations politiques présentent à leurs suffrages. Partant du principe «qu'être libre, c'est être bien informé ${ }^{23}$ et qu' «il importe [...] de connaître la provenance des ressources du parti politique et donc les influences et les pressions auxquelles il se trouvera exposé ${ }^{24}$, certains préconisent d'obliger les partis à publier un rapport périodique retraçant l'origine de leurs ressources. Marcel Waline juge de la sorte qu'il serait «élémentaire [...] que le chiffre effectif des cotisations [...] fût connu et publié » afin que «la République» ait

[...] la garantie que les décisions prises au nom des partis, et qui peuvent peser sur la politique du pays, représentent au moins l'expression libre et fidèle de cette minorité du corps électoral qui est embrigadée dans les organisations partisanes ${ }^{25}$.

Une autre raison avancée est la nécessité d'instaurer une certaine égalité entre les formations politiques dans la compétition politique ${ }^{26}$. Moins soucieux de préserver la liberté des partis que de les placer à égalité, ses partisans ajoutent à l'exigence de publication des comptes la fixation d'un plafond de financement privé, assorti, si besoin est, d'un financement public ${ }^{27}$, ce qui exige l'institutionnalisation d'un mécanisme de contrôle et de certification des comptes sophistiqué. Dégagées par Rainer Kraehe ${ }^{28}$, les premières raisons avancées par les tenants d'une politique d'encadrement des financements partisans - l'égalité des partis, l'information des citoyens sur les influences qui s'exercent sur eux, la sauvegarde de l'indépendance nationale, les risques de capture de l'État par des intérêts particuliers - sont différemment appréciées par la doctrine, qui se divise sur la façon de les articuler, sur leur conciliation avec la liberté des partis et les droits et libertés individuelles ${ }^{29}$, et par conséquent, sur les mécanismes de financement à adopter. Alors que la liberté des partis consacrée par la Constitution de 1958 rendait toute entreprise de codification hasardeuse, les propositions de régulation sont restées lettres mortes.

19. N. Tolini, Le financement des partis politiques, p. $47 s q$.

20. Sur ces projets, voir R. Kraehe, Le financement des partis politiques, p. 15; un premier modèle de statut est proposé dans F. Goguel (B. Serampuy), "Pour une réglementation des partis politiques», Esprit, n 80, 1939.

21. L'article 8, finalement abandonné lors de l'élaboration de la Déclaration des droits de 1946, prévoyait que «l'institution d'un parti unique est prohibée comme contraire aux principes de la liberté républicaine. Les électeurs et électrices fixent leur choix sur des candidats relevant des partis politiques légalement constitués. Ont seuls ce caractère les partis [...] qui: a) adhèrent aux principes posés par la Déclaration des droits de l'homme inscrite en tête de la Constitution; b) répondent par leur organisation interne à la règle démocratique; $c$ ) acceptent le contrôle par l'État de leurs ressources et de leurs dépenses». Sur ce point, voir N. Tolini, Le financement des partis politiques, p. 37.

22. M. Waline, Les partis contre la République, Paris, Éditions Rousseau et $C^{\mathrm{ie}}, 1948$, p. 93.

23. R. Kraehe, Le financement des partis politiques, p. 80.

24. Ibid., p. 79

25. M. Waline, Les partis contre la République, p. 93.

26. André Philip écrit en ce sens: «Il serait tout d'abord nécessaire d'assurer aux partis une véritable égalité, en organisant un contrôle public avec une publicité suffisante de leurs ressources financières» (A. Philip, «La crise de la démocratie parlementaire», Revue politique et parlementaire, novembre 1953, p. 225 sq.).

27. R. Kraehe, Le financement des partis politiques, p. 80.

28. Ibid.

29. Raymond Fusiller considérait par exemple que si les électeurs devaient être informés de l'origine des ressources des partis, la confidentialité du nom des donateurs devait être préservée pour éviter un tarissement du financement de certains partis (R. Fusiller, "Les finances des partis», Revue politique et parlementaire, octobre et novembre 1953, p. 141-161 et 258-276). 
De nouvelles raisons tirées des exigences du bon fonctionnement de la démocratie ont été avancées, dans les années 1970, en faveur d'un financement public ${ }^{30}$. Mais c'est seulement lorsqu'est apparue la nécessité d'obtenir des ressources suffisantes et pérennes à la suite des scandales de financements partisans de la fin des années 1980 qu'a été mise sur agenda une politique de régulation reposant sur deux problématisations. La volonté d'en finir avec les financements illégaux des partis qui exposaient des personnalités politiques de premier plan à d'importants risques judiciaires, provoquaient une perte de confiance des citoyens dans la politique, et accréditaient l'idée que le personnel politique était largement corrompu, a nourri une première problématisation minimaliste, qui, sur fond de moralisation des comportements politiques, est partie de l'idée que la démocratie ayant un coût, il fallait que l'État le supporte et accorde une aide publique. Le mécanisme retenu, après divers tâtonnements ${ }^{31}$, consiste à distribuer cette aide annuelle entre les partis qui ont présenté des candidats ayant obtenu au moins $1 \%$ des suffrages exprimés dans au moins cinquante circonscriptions lors du premier tour des élections législatives, proportionnellement au nombre de suffrages obtenus lors de ce premier tour, pour sa première fraction ${ }^{32}$, et en fonction du nombre de députés et de sénateurs déclarant se rattacher à chacun d'eux pour sa seconde. Cette contribution est complétée par une aide indirecte, faite d'une déduction fiscale sur les dons qui leur sont consentis par les personnes physiques, et permet aux formations électoralement trop faibles pour bénéficier de l'aide de ne pas être écartées de tout financement public ${ }^{33}$. Combiné avec le plafonnement des dépenses électorales et leur remboursement sous certaines conditions, cet apport d'argent public devait limiter les besoins financiers des partis et faire disparaitre les pratiques illégales de collecte de fonds.

Leur persistance a débouché sur l'élaboration d'une seconde problématisation beaucoup plus exigeante de la politique de régulation des financements partisans. Marquée par la volonté de lutter contre la corruption ${ }^{34}$ et la méfiance à l'égard des "puissances de l'argent ${ }^{35}$, elle repose sur l'idée qu'il faut soustraire les partis non seulement à l'influence des États étrangers, mais aussi à celle de certains intérêts particuliers pour éviter tout risque de capture, à travers eux, de l'action publique ${ }^{36}$. C'est ce paradigme renvoyant à l'idéal de la production de décideurs en apesanteur d'intérêt ${ }^{37}$ qui a justifié le plafonnement des dons des personnes physiques - 7500 euros par an à un même parti politique ${ }^{38}-$, l'interdiction de ceux des personnes morales, à l'exception des partis politiques et qui a justifié, par ce strict encadrement des financements privés et la disparition de l'apport des entreprises, la nécessité d'un financement public.

En l'absence d'une définition juridique des partis politiques et pour ne pas empiéter sur leur liberté, il a fallu pour mettre en place ces deux politiques - financement public et réglementation des dons - en produire une définition financière ${ }^{39}$; les partis sont les seules personnes morales autorisées à participer au financement des campagnes électorales et d'autres partis ${ }^{40}$ et doivent pour ce faire respecter un ensemble d'obligations financières: se doter d'une association de financement ou d'un mandataire financier afin de s'assurer de la régularité des fonds qu'ils reçoivent et emploient ${ }^{41}$, tenir une comptabilité

30. Valéry Giscard d'Estaing souhaite ainsi que «les partis politiques [...] soient assurés d'un financement public normal» (réunion de presse du 25 juillet 1974, citée par N. Tolini, Le financement des partis politiques, p. 192).

31. Voir Y. Poirmeur, D. Rosenberg, Droit des partis politiques, p. 160-161.

32. Sont aussi éligibles à cette fraction les partis qui n'ont présenté que des candidats dans une ou plusieurs collectivités territoriales relevant des articles 73 ou 74 de la Constitution ou en Nouvelle-Calédonie et dont les candidats ont obtenu chacun au moins $1 \%$ des suffrages exprimés dans l'ensemble des circonscriptions dans lesquelles ils se sont présentés.

33. Sur les conditions de conventionnalité des financements publics et les obligations imposées aux partis en matière financière, voir Cour EDH, 7 juin 2007, Parti national basque - organisation régionale d'Iparalde c. France, nº 71251/o1; Cour EDH, 10 mai 2012, Özgürlük Ve Dayanisma Partisi (ÖDP) c. Turquie, $\mathrm{n}^{\circ}$ 7819/o3; Cour EDH, 26 avril 2016, Cumhuriyet Halk Partisi c. Turquie, $\mathrm{n}^{\circ}$ 19920/13.

34. La loi no 92-122 du 29 janvier 1993 relative à la prévention de la corruption et à la transparence de la vie économique et des procédures publiques aborde ainsi dans son titre ${ }^{\text {er }}$ le financement des campagnes électorales et des partis politiques.

35. Le projet de loi présenté à l'automne 1992 par le gouvernement socialiste marque ce changement de paradigme: il proposait d'interdire les dons des personnes morales aux partis et aux candidats. Les parlementaires les maintiendront et la loi du 29 janvier 1993 s'en tiendra à leur plafonnement. Les comptes des partis devaient comporter la liste des entreprises en ayant effectué et leurs montants.

36. Cette problématisation est parfaitement synthétisée dans: Organisation de coopération et de développement économiques, Le financement de la démocratie. Financement des partis et des campagnes électorales et risque de capture de l'action publique, Paris, Éditions de l'OCDE (Examen de l'OCDE sur la gouvernance publique), 2017.

37. Sur sa construction, voir Y. Poirmeur, "L'homme politique et les bonnes mœurs. Construction et usages d'un répertoire de stigmates", in Les bonnes mours, J. Chevallier (dir.), Paris, Presses universitaires de France, 1994, p. 305-353.

38. Avant l'interdiction des dons des personnes morales, l'article 11-4 de la loi du 11 mars 1988 dans sa rédaction modifiée par l'article 13 de la loi du 15 janvier 1990 relative à la limitation des dépenses électorales et à la clarification du financement des activités politiques prévoyait un double plafonnement: «Les dons consentis par des personnes dûment identifiées à une ou plusieurs associations agréées en qualité d'association de financement ou à un ou plusieurs mandataires financiers d'un même parti politique ne peuvent annuellement excéder 50 ooo $\mathrm{F}$ s'ils émanent d'une personne physique et 500 ooo F s'ils émanent d'une personne morale».

39. CE, Ass., 30 octobre 1996, Élections municipales de Fos-sur-Mer, Revue française de droit administratif, 1997, concl. L. Touvet, p. 59 .

40. Art. 11-4 de la loi du 11 mars 1988, dans sa rédaction modifiée par l'art. 16 de la loi du 19 janvier 1995 relative au financement de la vie politique: «Les personnes morales à l'exception des partis ou groupements politiques ne peuvent contribuer au financement des partis ou groupements politiques, ni en consentant des dons, sous quelque forme que ce soit, à leurs associations de financement ou à leurs mandataires financiers, ni en leur fournissant des biens, services ou autres avantages directs ou indirects à des prix inférieurs à ceux qui sont habituellement pratiqués".

41. Art. 11 de la loi du 11 mars 1988, dans sa rédaction modifiée par la loi du 15 janvier 1990: «Les partis politiques et leurs organisations territoriales ou spécialisées qu'ils désignent à cet effet recueillent des fonds par l'intermédiaire d'un mandataire nommément désigné par eux, qui est soit une association de financement, soit une personne physique». 
retraçant leurs comptes et ceux des organismes, entreprises ou sociétés qui leur sont liés, les faire certifier, les déposer à la CNCCFP qui les rend publics. Les logiques qui nourrissent cette politique et visent à empêcher que des dons interdits servent à capturer l'action publique, que le bénéfice de l'aide publique directe ou indirecte soit obtenu frauduleusement, et au-delà que les fonds obtenus irrégulièrement faussent la compétition électorale en accroissant l'inégalité des chances entre formations politiques se sont heurtées à la garantie constitutionnelle de liberté des partis qui en a limité la portée. Les parlementaires qui souhaitaient préserver les marges d'action financière de leur parti ont laissé de nombreuses failles dans le dispositif de contrôle et la définition exclusivement financière du parti politique a favorisé l'apparition de nombreux micro-partis, sans militants ni adhérents. Repris et modélisé par les organes du Conseil de l'Europe qui en tirent les implications dans des lignes directrices et des recommandations ${ }^{42}$, le référentiel qui gouverne la politique de rationalisation des financements partisans en France, fondé sur le souci de lutter contre la corruption et d'éliminer tout risque de capture de l'action publique, est devenu, au fil du temps de plus en plus prégnant. Les lacunes et les défauts que comportaient la réglementation et les mécanismes de contrôle ont été peu à peu corrigés par des retouches législatives et des évolutions jurisprudentielles qui obéissent tendanciellement de plus en plus à ses logiques et accroissent la rationalité du dispositif.

\section{B. L'amélioration du dispositif d'encadrement, de contrôle et de sanctions}

La loi du 11 mars 1988 s'était bornée à exiger des partis bénéficiaires de l'aide publique qu'ils déposent leurs comptes à la fin de l'exercice aux bureaux des assemblées, qui les publiaient au Journal officiel de la République française $(J O R F)$. Ils n'étaient soumis à aucune analyse et les partis qui ne les transmettaient pas ne pouvaient plus bénéficier de l'aide. La légalisation de certains dons et leurs plafonnements a conduit le législateur à confier à un organe collégial, la CNCCFP, créée par la loi du 15 janvier 1990, le soin de délivrer l'agrément des associations de financement, de vérifier les comptes que les partis doivent produire, de les publier sommairement au JORF et de lister les formations éligibles à l'aide publique. Elle peut élaborer des recommandations sur la façon de présenter les comptes ainsi que des formulaires de présentation, et publie un rapport annuel qui lui permet sur la base d'une analyse de son activité de faire des suggestions pour améliorer le système de contrôle. Les insuffisances du contrôle qu'elle exerçait étaient patentes et tenaient d'abord au fait que le périmètre de consolidation et de certification des comptes ne comprenait pas nécessairement toutes les entités des partis et à l'impossibilité consécutive d'apprécier la légalité du financement des campagnes électorales ${ }^{43}$. L'article 11 de la loi de 1988, dans sa rédaction de la loi de 1990, restait flou sur la nature des fonds recueillis par l'intermédiaire de leur mandataire ${ }^{44}$.

Les faiblesses des vérifications résultaient aussi du flou, dans les textes initiaux, sur ce que recouvraient les dons des personnes physiques, sur l'absence d'indications sur le statut des cotisations des adhérents et des élus, ainsi que sur le régime des prêts, obtenus ou consentis par les partis. Les dons de personnes physiques étrangères, hormis leur plafonnement, et les emprunts auprès d'établissements financiers étrangers n'étaient pas encadrés. Plus largement, était posée la question de la pertinence du plafonnement des dons à 7500 euros par parti, qui encourageait la multiplication des micro-partis, et le contournement du principe du plafonnement lui-même par les micro-partis d'une même galaxie partisane. Sur tous ces points de sensibles améliorations sont intervenues récemment. Les modalités de versement des dons ont d'abord été précisées par l'article 5 de la loi de finance du 30 décembre 2005 qui prévoit que «[t] out don de plus de 150 euros [...] doit être versé, à titre définitif et sans contrepartie, soit par chèque, soit par virement, prélèvement automatique ou carte bancaire». L'article 15 de la loi du 11 octobre 2013 relative à la transparence de la vie publique redéfinit le régime des dons et cotisations. Désormais, «[l]es dons consentis et les cotisations versées en qualité d'adhérent d'un ou de plusieurs partis politiques par une personne physique dûment identifiée [...] ne peuvent annuellement excéder 7500 euros ». Mais, « [p] ar exception, les cotisations versées par les titulaires de mandats électifs nationaux ou locaux ne sont pas prises en compte pour le calcul [de ce] plafond ${ }^{45}$. Cette mesure destinée, entre autre, à lutter contre les micro-partis, a été assortie d'une obligation de transparence: les partis sont tenus de communiquer chaque année, dans les conditions prévues par un décret, la liste des personnes ayant consenti à verser un ou plusieurs dons ou cotisations avec indication de leur montant à la CNCCFP ${ }^{46}$. Si l'interdiction des contributions et aides matérielles d'un État étranger ou d'une personne morale de droit

42. Commission européenne pour la démocratie par le droit, Code de bonne conduite... et Rapport explicatif, $\mathrm{n}^{\circ}{ }_{154}, \mathrm{p} .42$.

43. Pour un inventaire de ces problèmes, voir Y. Poirmeur, D. Rosenberg, Droit des partis politiques, p. 172-173; pour une actualisation de ces insuffisances, voir J.-F. Kerléo, "Le périmètre comptable des partis politiques», in Financement et moralisation de la vie politique, E. Forey, A. Granero, A. Meyer (dir.), Paris, Institut universitaire Varenne, 2018, p. 67-81.

44. «Les partis politiques et leurs organisations territoriales ou spécialisées qu'ils désignent à cet effet recueillent des fonds par l'intermédiaire d'un mandataire nommément désigné par eux, qui est soit une association de financement, soit une personne physique».

45. Sur les modalités de ces versements, voir C. Oriol, «Versement direct à un parti de l'indemnité due à un élu. Comment une commune se trouve piégée par sa méconnaissance des textes légaux», L'actualité juridique. Droit administratif, 2017, p. 2414.

46. Cette loi - art. 11 - a en outre interdit aux parlementaires qui ne sont pas élus dans une circonscription d'outre-mer de s'inscrire ou de se rattacher à un parti ou un groupement politique ultra-marin. Voir J.-P. Camby, "Mandat parlementaire et financement des partis politiques", Petites affiches, $\mathrm{n}^{\circ} 179,8$ septembre 2014, p. 5. 
étranger avait été initialement posée par l'article 13 de la loi du 15 janvier 1990 relative à la limitation des dépenses électorales et à la clarification du financement des activités politiques, les dons des personnes physiques étrangères étaient possibles. L'article 25 de loi du 15 septembre 2017 pour la confiance dans la vie politique vient enfin de limiter le versement de dons aux seules personnes physiques qui sont de nationalité française ou qui résident en France. Encadrant les prêts, il limite aux "partis et groupements politiques ainsi qu['aux] établissements de crédit et sociétés de financement ayant leur siège social dans un État membre de l'Union européenne ou partie à l'accord sur l'Espace économique européen » la possibilité de leur en consentir ou d'apporter leur garantie aux prêts qui leur sont octroyés. Il est expressément prévu qu'ils ne peuvent recevoir de prêts d'un État étranger ou d'une personne morale de droit étranger, "à l'exception des établissements de crédit ou sociétés de financement» qui satisfont aux conditions de localisation de leur siège social pour pouvoir leur en octroyer ${ }^{47}$.

Parallèlement, le contenu de la comptabilité à transmettre a été substantiellement complété. Alors que depuis 1990 l'article 11-7 de la loi de 1988 prévoyait seulement qu'elle devait

[...] retracer tant les comptes du parti ou groupement politique que ceux de tous les organismes, sociétés ou entreprises dans lesquels le parti ou groupement détient la moitié du capital social ou des sièges de l'organe d'administration ou exerce un pouvoir prépondérant de décision ou de gestion ${ }^{4}$,

la loi du 6 mars 2017 tendant à renforcer les obligations comptables des partis politiques et des candidats a élargi l'objet de la transmission qui doit comporter

[...] dans les annexes de ces comptes, les montants et les conditions d'octroi des emprunts souscrits ou consentis par eux, l'identité des prêteurs ainsi que les flux financiers avec les candidats tenus d'établir un compte de campagne ${ }^{49}$.

La publication des comptes, qui n'est plus sommaire, mais détaillée ${ }^{50}$, comprend désormais l'indication «des montants consolidés des emprunts souscrits répartis par catégories de prêteurs, types de prêts et par pays d'établissement ou de résidence des prêteurs, ainsi que l'identité des prêteurs personnes morales et les flux financiers nets avec les candidats ${ }^{51}$. Achevant ce mouvement de consolidation des comptes certifiés, la loi du 15 septembre 2017 pour la confiance dans la vie politique (art. 25 (V)) précise que la comptabilité est tenue selon un règlement établi par l'Autorité des normes comptables et étend son périmètre de consolidation qui «inclut les comptes des organisations territoriales du parti ou groupement politique dans des conditions définies par décret ${ }^{52}$. Une nouvelle rédaction de l'article 11 de la loi de 1988 par la loi du 15 septembre 2017 a enfin imposé que «l'ensemble des ressources, y compris les aides» des partis et de leurs organisations territoriales et spécialisées qu'ils désignent à cet effet sont recueillies par l'intermédiaire d'un mandataire. Cette mesure comble une importante lacune dans la consolidation et la traçabilité des ressources, puisque jusqu'alors seuls les dons et les cotisations ouvrant droit au bénéfice de la réduction d'impôt devaient lui être versées ${ }^{53}$.

Les pouvoirs de contrôle de la CNCCFP ont été également renforcés à plusieurs reprises. L'article 17 de la loi du 11 octobre 2013 relative à la transparence de la vie publique lui a donné la possibilité de «demander le cas échéant, communication de toutes les pièces comptables et de tous les justificatifs nécessaires au bon accomplissement de sa mission de contrôle». De plus, alors qu'en cas de manquement aux obligations comptables de l'article 11-7 de la loi du 11 mars 1988 il était seulement prévu que «le parti ou groupement politique perd le droit, pour l'année suivante, au bénéfice des dispositions des articles 8 à 10 de la présente loi », l'article 17 de la loi du 11 octobre 2013 en a explicité les conséquences pour les dons et cotisations à son profit qui «ne peuvent, à compter de l'année suivante, ouvrir droit à la réduction d'impôt» pour les dons et cotisations. La loi du 6 mars 2017 a précisé le rôle de la CNCCFP dans cette opération, «sans modifie[r] le régime de sanction ${ }^{54}$, en indiquant qu'elle peut, si elle constate un manquement, "priver, pour une durée maximale de trois ans» de ces avantages. Pour autant, si la CNCCFP peut mieux appréhender les comptes, le renforcement de leur contrôle se heurte toujours au respect de la liberté constitutionnellement garantie des partis. Son intervention, encore trop formelle, se restreint au constat de leur «incohérence manifeste ${ }^{55}$. Au-delà,

47. Ce durcissement est une réponse à la question des prêts russes du Front national et aux risques de contournement de la réglementation des dons que la multiplication des prêts comportait; voir R. Rambaud, "Confiance dans la vie politique: la révolution attendra... », L'actualité juridique. Droit administratif, 2017, p. 2237.

48. Art. 11-7 de la loi de 1988, dans sa rédaction modifiée par l'art. 13 de la loi de 1990.

49. Art. L. 52-12 du Code électoral.

50. Voir R. Rambaud, «Confiance dans la vie politique... », p. 2237

51. Art. 11-7 de la loi de 1988, dans sa version du 6 mars 2017.

52. La loi du 6 mars 2017 tendant à renforcer les obligations comptables des partis politiques et des candidats (art. 7) a modulé le processus de certification: les partis dont les ressources annuelles ne dépassent pas 230000 euros peuvent désormais être certifiés par un seul commissaire aux comptes, au lieu de deux.

53. Les autres cotisations pouvaient être perçues directement par le trésorier du parti; voir CNCCFP, Dix-huitième rapport d'activité, 2016, Paris, Direction de l'information légale et administrative, 2017, p. 55-56.

54. CE, 12 juin 2017, Avis sur un projet de loi pour la confiance dans l'action publique, en ligne: http://www.conseil-etat.fr/content/download/102779/1027456/ version/1/file/Avis\%2on393324.pdf, p. 7, nº 27.

55. CE, 9 juin 2010, Association Cap sur l'avenir 13: la CNCCFP «ne saurait [...] sans excéder sa compétence, constater, en l'absence de toute incohérence manifeste, qu'un parti [...] a manqué à ses obligations, alors même qu'il a déposé en temps utile des comptes certifiés correspondant 
les sanctions pénales ont progressivement été durcies et adaptées à l'évolution du régime des dons et des prêts ${ }^{56}$ et aux violations des obligations de communication d'informations financières à la CNCCFP ${ }^{57}$. Malgré ces avancées législatives significatives ${ }^{5}$, l'argent des partis paraît toujours «insuffisamment surveillé $»^{59}$, qu'il s'agisse des recettes et surtout des dépenses ${ }^{60}$. Pour inaboutie qu'elle soit, cette stratégie de régulation des financements partisans a sensiblement modifié la condition financière des partis, dont il convient d'apprécier les conséquences.

\section{Les incidences du système de financement public}

Dominés par le souci de soustraire les partis à l'influence de l'argent privé, l'interdiction complète des dons de personnes morales, le plafonnement de ceux des personnes physiques, incluant les cotisations, versées à un ou plusieurs partis, l'encadrement des prêts qu'ils sollicitent, l'instauration d'un financement public et le remboursement aux candidats d'une partie de leurs dépenses de campagne ont bouleversé l'économie des financements partisans $^{61}$ et les formes de pression auxquelles ils sont soumis (A). Le financement public est devenu, sur période longue, une source principale de revenu, devant les cotisations des élus et des adhérents, pour les grands partis qui en sont les principaux bénéficiaires ${ }^{62}$, tandis qu'ils connaissaient de substantielles transformations organisationnelles, les rapprochant du modèle du parti cartel qui tire l'essentiel des ressources nécessaires à ses activités de l'État, parvient ainsi à se stabiliser durablement dans les positions de pouvoir et devient une agence para étatique ${ }^{63}$. Par ailleurs, les micro-partis se sont multipliés - 451 partis devaient déposer leurs comptes auprès de la CNCCFP en $2015^{64}$-, ce qui est considérable même dans un pays où la volatilité des étiquettes est forte ${ }^{65}$. Ce sont ainsi des mutations de la forme parti qu'induirait le changement de leur condition financière (B).

\section{A. La soustraction des partis aux pressions financières}

Même si on peut relever une étatisation du financement ${ }^{66}$ des partis qui bénéficient de l'aide publique ${ }^{67}$, plus nette encore lorsqu'on y ajoute les cotisations versées par les élus sur leurs indemnités, on ne saurait conclure pour autant à une étatisation des partis. Plusieurs raisons peuvent à cet égard être avancées. Outre le fait qu'ils demeurent des organismes de droit privé ${ }^{68}$ - des associations à statut spécial-, dont la liberté est constitutionnellement protégée, les contraintes qui leur sont imposées en contrepartie de l'aide demeurent très limitées. La modulation paritaire,

au périmètre fixé par la loi, que cette certification soit établie sans réserves ou qu'elle soit assortie de réserves, même formulées sur des points identiques plusieurs années de suite"; voir aussi CE, 12 juin 2017, Avis sur un projet de loi pour la confiance dans l'action publique, p. 7.

56. Art. 11-5 de la loi de 1988 modifié par la loi du 15 septembre 2017: «Les personnes qui ont versé un don ou consenti un prêt à un ou plusieurs partis ou groupements politiques en violation des articles 11-3-1 et 11-4 sont punies de trois ans d'emprisonnement et de 45 ooo $€$ d'amende. Les mêmes peines sont applicables au bénéficiaire du don ou du prêt consenti: $1^{\circ}$ Par une personne physique en violation de l'article 11-3-1 et du cinquième alinéa de l'article $11-4 ; 2^{\circ}$ Par une même personne physique à un seul parti ou groupement politique en violation du premier alinéa du même article $11-4 ; 3^{\circ}$ Par une personne morale, y compris de droit étranger, en violation dudit article 11-4».

57. Art. 11-9 de la loi de 1988 modifié par la loi du 15 septembre 2017: «I - Le fait de ne pas communiquer, de sa propre initiative ou sur la demande de la CNCCFP, les informations qu'un parti ou groupement politique est tenu de communiquer à cette dernière en application de l'article 11-3-1, du quatrième alinéa de l'article 11-4 et du II de l'article 11-7 est puni d'un an d'emprisonnement et de $15000 €$ d'amende. II - Le fait pour un dirigeant de droit ou de fait d'un parti ou groupement politique de ne pas déposer les comptes du parti ou groupement qu'il dirige dans les conditions fixées à l'article 11-7 est puni de trois ans d'emprisonnement et de $45000 €$ d'amende».

58. Le Conseil d'État a estimé contraire à la liberté des partis de vouloir leur imposer des règles d'engagement et d'exécution des dépenses, l'instauration d'une certification des comptes par la Cour des comptes lorsque leurs ressources annuelles dépassent 500 ooo euros; voir CE, 12 juin 2017 , Avis sur un projet de loi pour la confiance dans l'action publique, p. 6.

59. R. Rambaud, «L'argent et les partis», Pouvoirs, $n^{\circ} 163,2017$, p. 87 sq.

60. Des factures de plusieurs millions d'euros auraient été payées par l'Union pour un mouvement populaire (UMP) à la société Bygmalion pour couvrir les dépenses de la campagne présidentielle de Nicolas Sarkozy en 2012, qui dépassaient les plafonds autorisés; voir L. Equy, S. Mouillard, D. Albertini, «Fausses factures, Bygmalion, Sarkozy: le grand déballage», Libération, 26 mai 2014.

61. Y. Poirmeur, D. Rosenberg, Droit des partis politiques, p. 174.

62. Ainsi de 2012 à 2017, cinq partis sur les treize éligibles percevaient plus de $86 \%$ de l'aide versée, dont $69 \%$ pour le Parti socialiste et Les Républicains; voir CNCCFP, Dix-huitième rapport d'activité, 2016, p. 65-66. Pour une analyse de la période 1993-2012, voir A. François, É. Phélippeau, Le financement de la vie politique. Réglementations, pratiques et effets politiques, Paris, A. Colin, 2015, p. 61.

63. Voir R. S. Katz, P. Mair, "La transformation des modèles d'organisation et de démocratie dans les partis. L'émergence du parti-cartel», in Les systèmes de partis dans les démocraties occidentales: le modèle du parti-cartel en question, Y. Aucante, A. Dézé (dir.), Paris, Presses de Sciences Po, 2008, p. 49-50.

64. Ils étaient 28 en 1990, 161 en 1995, 208 en 2000, 259 en 2005, 296 en 2010 et 451 en 2015 (sources: rapports d'activité de la CNCCFP)

65. Y. Poirmeur, Les partis politiques..., p. 9 .

66. J.-L. Debré, «Intervention", Journal officiel de l'Assemblée nationale, Débats, 13 octobre 1992, p. 360.

67. Cette aide représentait, au titre de l'exercice 2015, $45 \%$ de ses ressources pour le Parti socialiste, $51 \%$ pour Les Républicains, $68 \%$ pour le Parti radical de gauche, 36 \% pour le Front national, 65 \% pour le Nouveau Centre, 36 \% pour Europe Écologie Les Verts, 36 \% pour Debout la France et 10 \% pour le Parti communiste français; voir R. Rambaud, «L'argent et les partis», p. 92. Sur sa part en 2011, voir Y. Poirmeur, Les partis politiques..., p. 154.

68. Confirmé par Cass., $1^{\text {re }}$ civ., 25 janvier 2017, Association Front national c. J.-M. X, $\mathrm{n}^{\circ} 102$ : «Si les partis et groupements politiques concourent à l'expression du suffrage et jouent un rôle essentiel au bon fonctionnement de la démocratie, le principe de liberté de formation et d'exercice qui leur est constitutionnellement garanti s'oppose à ce que les objectifs qu'ils poursuivent soient définis par l'administration et à ce que le respect de ces objectifs soit soumis à son contrôle, de sorte qu'ils ne sauraient être regardés comme investis d'une mission de service public». 
afin de favoriser l'égal accès des femmes et des hommes aux mandats électoraux, et les obligations comptables n'empiètent guère sur leur liberté et n'ont pas eu de conséquence, si l'on en juge par le vaste spectre des idéologies qui se manifestent dans l'espace politique ${ }^{69}$ et par l'éclosion d'une myriade de micro-partis. Cette étatisation des financements partisans, qui permet aux grands partis de disposer de subsides garantis et suffisants, s'effectue dans un cadre juridique qui sauvegarde le pluralisme politique $^{70}$ : elle n'empêche ni l'entrée d'outsiders dans la compétition politique - ce qu'illustre le succès à l'élection présidentielle et aux élections législatives de 2017 d'un tout nouveau parti, La République en marche ${ }^{71}$, face aux candidats de grands partis qui concentraient depuis des années l'aide publique -, ni n'assure les grands partis qui disposent de ressources pérennes pendant la législature qu'ils pourront ensuite s'y maintenir durablement ${ }^{72}$. En tout état de cause, si dépendance à l'État des organisations partisanes il y avait à travers l'aide publique, force serait de constater qu'elle serait variable, puisque, à montant égal, son importance relative est fonction de la capacité différentielle de chacune à se procurer d'autres ressources. Enfin, cette dépendance n'est pas corroborée par «l'effervescence partisane ${ }^{73}$ des petits et des micro-partis. Si l'aide publique, à laquelle assez peu d'entre eux sont éligibles $^{74}$, et les déductions fiscales sur les dons et cotisations contribuent sans doute à leur multiplication, c'est surtout l'interdiction faite aux personnes morales autres que des partis de financer les campagnes électorales ${ }^{75}$ qui incite tous ceux qui souhaitent intervenir dans la vie politique, notamment être candidat à une élection nationale ou locale, à se doter d'une telle enveloppe juridique, dont la création est très peu exigeante.

L'impossibilité pour les personnes morales de financer légalement les partis en raison «du risque réel que certains partis et candidats, une fois au pouvoir, soient plus réceptifs aux intérêts d'un groupe particulier de

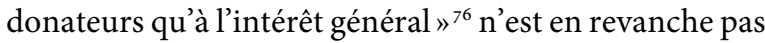
sans conséquences. Elle contribue à affaiblir le lien des partis avec la société civile dont les groupes d'intérêt et les entreprises de toute nature sont des acteurs essentiels. Outre les contournements qu'il est difficile d'éliminer complètement, cette législation génère des effets de déplacement. L'interdiction du financement des partis par les personnes morales a conduit ces acteurs à réorienter leurs stratégies d'influence en consacrant davantage d'argent aux différentes activités de lobbying ${ }^{77}$, notamment des campagnes de lobbying électoral ${ }^{78}$, grâce auxquelles ils cherchent à se faire entendre et à peser tout à la fois sur les électeurs, sur les partis, et sur les candidats ${ }^{79}$. Quoi qu'il en soit, la législation sur les financements partisans gouvernée par le souci de limiter l'influence des intérêts économiques et financiers et de placer les partis et leurs élus en apesanteur d'intérêts ${ }^{80}$ n'a fait qu'accentuer leur éloignement de la société civile et favoriser la mutation de la forme parti.

\section{B. Les métamorphoses des partis}

C'est moins la structure du champ politique, que l'organisation des partis que le système de financement public affecte. Il amplifie les évolutions qui touchent les partis et induisent le changement de leur forme en les rapprochant de celle du parti cartel. Le financement public permet ainsi à ses principaux bénéficiaires, frappés par le déclin du militantisme, sans grande capacité de mobilisation ${ }^{81}$ et disposant de peu de militants, d'assurer leurs fonctions partisanes - sélection des candidats, fonction programmatique, communication et marketing politique... - en les confiant à une administration bien structurée et de plus en plus professionnalisée et / ou en les sous-traitant à

69. Voir M. Offerlé, Sociologie de la vie politique française, Paris, La Découverte, 2004, p. 36-37.

70. Voir Y. Poirmeur, "La réglementation du financement des partis politiques en France: la consécration du parti cartel? ", in Penser la science administrative dans la post-modernité: mélanges en l'honneur du professeur Jacques Chevallier, P. Bezes, M. Borgetto, D. Bourcier (dir.), Paris, LGDJ, 2013, p. 185

71. Le temps de parole sur les chaînes du service public a été ajusté pour tenir compte du renouvellement du paysage politique; voir S. Salles, «L'adaptation de la législation électorale par le Conseil constitutionnel: plus de temps pour "En marche !" ", Gazette du Palais, n²5, 4 juillet 2017, p. 33.

72. Le Parti socialiste et Les Républicains ont connu récemment d'importants revers de fortunes. La perte d'élus - donc de cotisations - et la diminution de l'aide publique aggravent leurs difficultés financières et les contraignent à vendre leur siège parisien et les locaux de leurs permanences lorsqu'ils en sont propriétaires. L'argent ne fait pas tout en matière électorale, comme le montrent les déconvenues de Nicolas Sarkozy à la présidentielle de 2012, où il aurait dépensé le double du plafond autorisé. Au total, près de 46 millions auraient été utilisés; voir M. Mathieu, «Sarkozy a dépensé le double du plafond autorisé», Mediapart, 13 avril 2016.

73. A. François, É. Phélippeau, Le financement de la vie politique..., p. 72.

74. Pour la législature 1988-1993, ils étaient 16 à l'être, 47 pour celle de 1993-1997, 57 pour celle de 1997-2002, 70 pour celle de 2002-2007, 54 pour celle de 2007-2012, et 52 pour celle de 2012-2017; voir A. François, É. Phélippeau, Le financement de la vie politique..., p. 72.

75. Pour une analyse de la jurisprudence et de la portée de cette règle, voir E. Forey, «Une association proche d'un parti politique peut-elle contribuer au financement d'une campagne électorale?", L'actualité juridique. Droit administratif, 2016, p. 440.

76. Organisation de coopération et de développement économiques, Le financement de la démocratie..., p. 22.

77. Sur son développement, voir Y. Poirmeur, Lobbying et stratégies d'influences. Du XVIII ${ }^{e}$ au XXIe siècle en France, Issy-les-Moulineaux, LGDJ, 2018.

78. Voir Le lobbying électoral: groupes en campagne présidentielle (2012), G. Courty, J. Gervais (dir.), Villeneuve d’Ascq, Presses universitaires du Septentrion, 2016.

79. La logique de la législation sur les partis devrait conduire à réglementer étroitement le financement de ces campagnes de communication d'influence.

80. Voir Y. Poirmeur, «La transparence dans le discours politique actuel », in La transparence en politique, N. Droin, E. Forey (dir.), Bayonne - Paris, Institut universitaire Varenne - LGDJ, 2013, p. 106.

81. Voir J. Fretel, R. Lefebvre, «La faiblesse des partis politiques français: retour sur un lieu commun historiographique», in La fabrique interdisciplinaire: histoire et science politique, M. Offerlé, H. Rousso (dir.), Rennes, Presses universitaires de Rennes, 2008, p. 154. 
des agences spécialisées et à des consultants ${ }^{82}$, en utilisant des techniques de communication modernes et, avec plus ou moins de succès ${ }^{83}$, en externalisant, par le procédé des primaires ouvertes, la sélection de leurs candidats aux élections auxquels est transférée la fonction programmatique $^{84}$, ce qui permet tout à la fois de trancher des concurrences internes, de mobiliser leurs sympathisants et de les rapprocher de la société civile, en principe, d'optimiser le choix de leurs candidats et de pré-mobiliser leur électorat ${ }^{85}$. Ces procédures participatives, contrôlées par les dirigeants qui maîtrisent la production des offres politiques, ne contrebalancent pas les logiques oligarchiques ${ }^{86}$ à l'œuvre dans ces organisations qui, converties en partis électoraux professionnels, privilégient l'activité électorale, réduisent le rôle des militants, s'éloignent de la société civile et confèrent à leur direction une grande autonomie. Cette métamorphose qui accentue leurs défauts ne saurait, dans ces conditions, restaurer l'image des partis dans l'opinion.

82. Voir Y. Poirmeur, Les partis politiques..., p. 164 sq.

83. Sur la conversion aux primaires, voir R. Lefebvre, É. Treille, «Vers une primarisation de la vie politique française?», in Les primaires ouvertes en France. Adoption, codification, mobilisation, R. Lefebvre, É. Treille (dir.), Rennes, Presses universitaires de Rennes, 2016, p. 11 sq.

84. Pour Rémi Lefebvre, «la fonction programmatique est dès lors en quelque sorte privatisée (chaque candidat fabrique un projet qui justifie sa candidature)», ce qui «contribue aussi à dévaluer le militantisme», car les militants sont dépossédés de la production collective du projet et de la «gratification symbolique qu'est le pouvoir d'investiture». Leur rôle «se réduit désormais à la [...] fonction d'organisation de la procédure des primaires» (R. Lefebvre, "Les primaires ouvertes et les partis politiques», Revue du droit public, n 3, 2017, p. 515 sq.).

85. Sur leur financement et l'imputation des dépenses au compte du candidat désigné, voir R. Rambaud, «Le financement de la vie politique et les primaires ouvertes en France», in Les primaires ouvertes en France..., p. 125 sq.

86. Sur la loi d'airain de l'oligarchie, voir R. Michels, Les partis politiques: essai sur les tendances oligarchiques des démocraties, Paris, Flammarion, 1971. 